\title{
Analytical Study of the Impact of the Mobility Node on the Multi-channel MAC Coordination Scheme of the IEEE 1609.4 Standard
}

\author{
Doan Perdana ${ }^{1}$, Ray-Guang Cheng ${ }^{2}$, Riri Fitri Sari ${ }^{3}$ \\ ${ }^{1}$ Telecommunication Engineering, School of Electrical Engineering, Telkom University, Bandung City- Indonesia, \\ ${ }^{2}$ Department of Electronics and Computer Engineering, National Taiwan University of Science and Technology, \\ Taipei-Taiwan, ${ }^{3}$ Department of Electrical Engineering, Faculty of Engineering, University of Indonesia, Depok - \\ Indonesia \\ [e-mail: doanperdana@telkomuniversity.ac.id, crg@mail.ntust.edu.tw, riri@ui.ac.id] \\ *Corresponding author: Doan Perdana
}

Received September 9, 2016; accepted November 25, 2016; published January 31, 2017

\begin{abstract}
The most challenging issues in the multi-channel MAC of the IEEE 1609.4 standard is how to handle the dynamic vehicular traffic condition with a high mobility, dynamic topology, and a trajectory change. Therefore, dynamic channel coordination schemes between $\mathrm{CCH}$ and SCH are required to provide the proper bandwidth for $\mathrm{CCH} / \mathrm{SCH}$ intervals and to improve the quality of service (QoS). In this paper, we use a Markov model to optimize the interval based on the dynamic vehicular traffic condition with high mobility nodes in the multi-channel MAC of the IEEE 1609.4 standard. We evaluate the performance of the three-dimensional Markov chain based on the Poisson distribution for the node distribution and velocity. We also evaluate the additive white Gaussian noise (AWGN) effect for the multi-channel MAC coordination scheme of the IEEE 1609.4 standard. The result of simulation proves that the performance of the dynamic channel coordination scheme is affected by the high node mobility and the AWGN. In this research, we evaluate the model analytically for the average delay on CCHs and SCHs and also the saturated throughput on SCHs.
\end{abstract}

Keywords: MAC Multichannel, IEEE 1609.4, Dynamic channel coordination scheme, $\mathrm{CCH} / \mathrm{SCH}$ interval, Markov chain three Dimensional. 


\section{Introduction}

The vehicular ad hoc network (VANET) is an outcome of wireless technology progress in MANET. The VANET is regarded as the most appropriate network to use currently and in the future because it generates a unified vehicle strategy to resolve the traffic condition when crossing the street in a city.

IEEE 1609.4 specifies the extension to the IEEE 802.11p medium access control (MAC) for multi-channel operations [1]. According to standard, there is one control channel (CCH) and multiple service channels (SCHs). The safety and control messages are exchanged between devices in the $\mathrm{CCH}$. The non-safety application services are exchanged in the SCHs [1]. According to the coordination scheme, each device should alternate between the $\mathrm{CCH}$ and SCHs [1]. The IEEE 1609.4 standard defines a concept of channel intervals in which frequency is divided into one CCH and six SCHs [2]. The IEEE 1609.4 standard characterizes the synchronization interval with a fixed length of $100 \mathrm{~ms}$, which are divided into $\mathrm{CCH}$ and SCH intervals, i.e., each interval lasts for $50 \mathrm{~ms}$ [2]. The IEEE standard of 1609.4 synchronizes the $\mathrm{CCH}$ and $\mathrm{SCH}$ intervals to an external time reference, a global positioning system provide the universal coordinated time (UTC) [1].

In the IEEE 1609.4 specification, a WAVE provider, which could either be a roadside unit or vehicle, can initialize a Basic Service set (BSS) to provide a non-safety service [1]. Each WAVE provider advertises its presence and offers services by periodically broadcasting a WAVE service advertisement (WSA)

WSAs contain the data of the offered administrations and the system parameters important to join the promoted BSS (distinguishing proof, SCH, EDCA parameter sets, Internet arrangement, and so forth.) [1]. The IEEE 1609.4 standard suggests that each WAVE provider sends WSAs several times in the CCH interval [1]. A WAVE provider should also choose the least congested SCH for its BSS to reduce interference between nearby BSSs [1].

The results from literature show that fixed-length $\mathrm{CCH} / \mathrm{SCH}$ intervals cannot effectively handle a changing traffic load dynamically [1]. In a dynamic vehicular traffic condition, a fixed-length $\mathrm{CCH}$ can not handle the big number of safety and packets control [1]. For example, the $\mathrm{CCH}$ channel is rarely used because of occasional transmissions from vehicles in scanty systems [1], while certain applications expend a lot of data transfer capacity, for example, video downloads and outline, and can't acquire adequate SCH assets because of exorbitant conflict [1]. In this way, many protocols have been aim to address a dynamic setting of CCH/SCH intervals [1] to optimize utilization of channel and improve the quality of service (QoS).

A recent study that investigated the dynamic channel coordination scheme describes a system parameter in their algorithm to analyse $\mathrm{CCH} / \mathrm{SCH}$ intervals [1]. If the system parameter is not adjusted according to the dynamically changing network condition, the operation of the protocols cannot be guaranteed to work accurately under the dynamic traffic vehicular environment [1]. Furthermore, the authors used a fixed rate parameter in their algorithm to calculate the $\mathrm{CCH} / \mathrm{SCH}$ interval using the default option of a typical VANET system. 


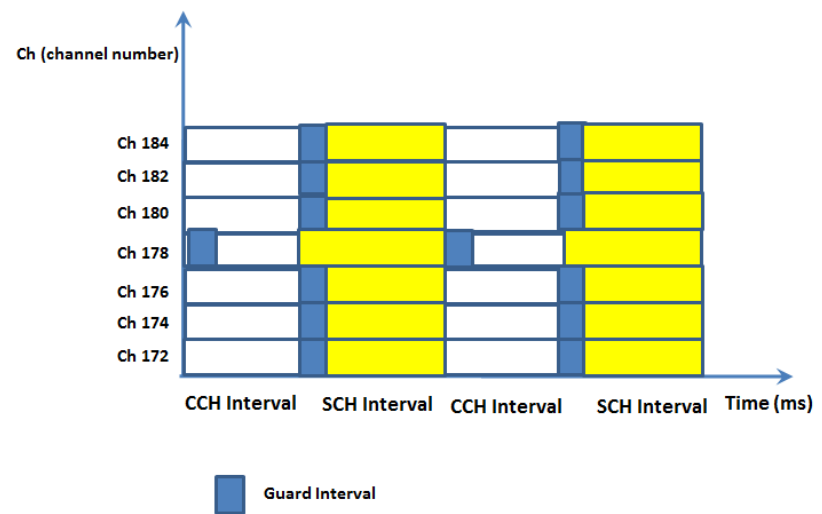

Fig. 1. The channel interval structure based on IEEE 1609.4 standard [1]

In this paper, we evaluate the performance of the dynamic channel coordination scheme under a dynamic vehicular traffic condition with high mobility nodes in the multi-channel MAC of the IEEE 1609.4 standard. We use a Markov chain model based on the Poisson distribution for node distribution and velocity to optimize and validate the analytical model of the WSA interval, safety interval, SCH interval, and also the saturated throughput on the $\mathrm{CCH}$ and SCHs interval delay. We also evaluate the Doppler effect impact on the proposed dynamic coordination scheme.

This research is created as follows. In section II, we develop a number of related works. Section III, the overall system model for a system supporting the dynamic channel coordination scheme is defined and then analysed using a Markov chain. In section IV, we evaluate a performance. Finally, section V shows the conclusions and suggests future work.

\section{Related Work}

Evaluating dynamic adjustments of CCH and SCH intervals in how they handle a congested vehicular traffic condition is essential when discussing a multi-channel MAC of the IEEE 1609.4 standard. Wang, Q. et al. [3-5] categorized the multi-channel MAC protocol into CCH and SCH intervals. Moreover, the scheme [3-5] divides the CCH interval into the safety and WSA interval. This scheme is called the variable CCH interval (VCI). VCI optimizes the ratio between the CCH and SCH intervals [3-5] to handle the dynamic vehicular traffic condition. We used [3-5] to evaluate the performance of the proposed scheme using a three-dimensional Markov chain based on the Poisson distribution for node distribution and velocity in a multi-channel MAC of the IEEE 1609.4 standard.

In [6], J. So et al. proposed a DSRC MAC protocol to give multi-channel performance. The focus offers potentially big bandwidth to non-safety implementation provided by the roadside manufacture that does not compromise safety communication in other channels. This approach supplements the current impromptu plans when a RSU is inaccessible. In any case, every gadget must be furnished with an alternate convention in both the impromptu mode and framework mode in the MAC and system layers. Thusly, a multifaceted nature of the gadget execution incredibly increments.

Differently, D. Zhu et al. [7] proposed a multi-channel MAC convention for the dynamic change of $\mathrm{CCH} / \mathrm{SCH}$ interims, called the element $\mathrm{CCH}$ interim (DCI). DCI works 
indistinguishably to VCI aside from how the WSA interim is figured. DCI ascertains the ideal WSA interim in light of the likelihood dissemination of the reservation time for an administration bundle in the $\mathrm{CCH}$ interim.

N. Lu et al. [8] characterized the devoted multi-channel MAC (DMMAC) to play out a versatile telecom system to diminish the crash rate and transmission delay. The objective of the exploration was to enhance the security execution of the parcel conveyance proportion. The examination had not reason an adaptive modification of a $\mathrm{CCH}$ interim; likewise, explanatory research were doered on the model of the aim conspire.

Most of works that study the coordination scheme in a multi-channel MAC of the IEEE 1609.4 standard did not evaluate the effect of a high mobility node and frame error caused by AWGN. This motivated us to evaluate the coordination scheme under the dynamic vehicular traffic condition with high mobility nodes in the multi-channel MAC of the IEEE 1609.4 standard.

\section{Modelling and Analysis}

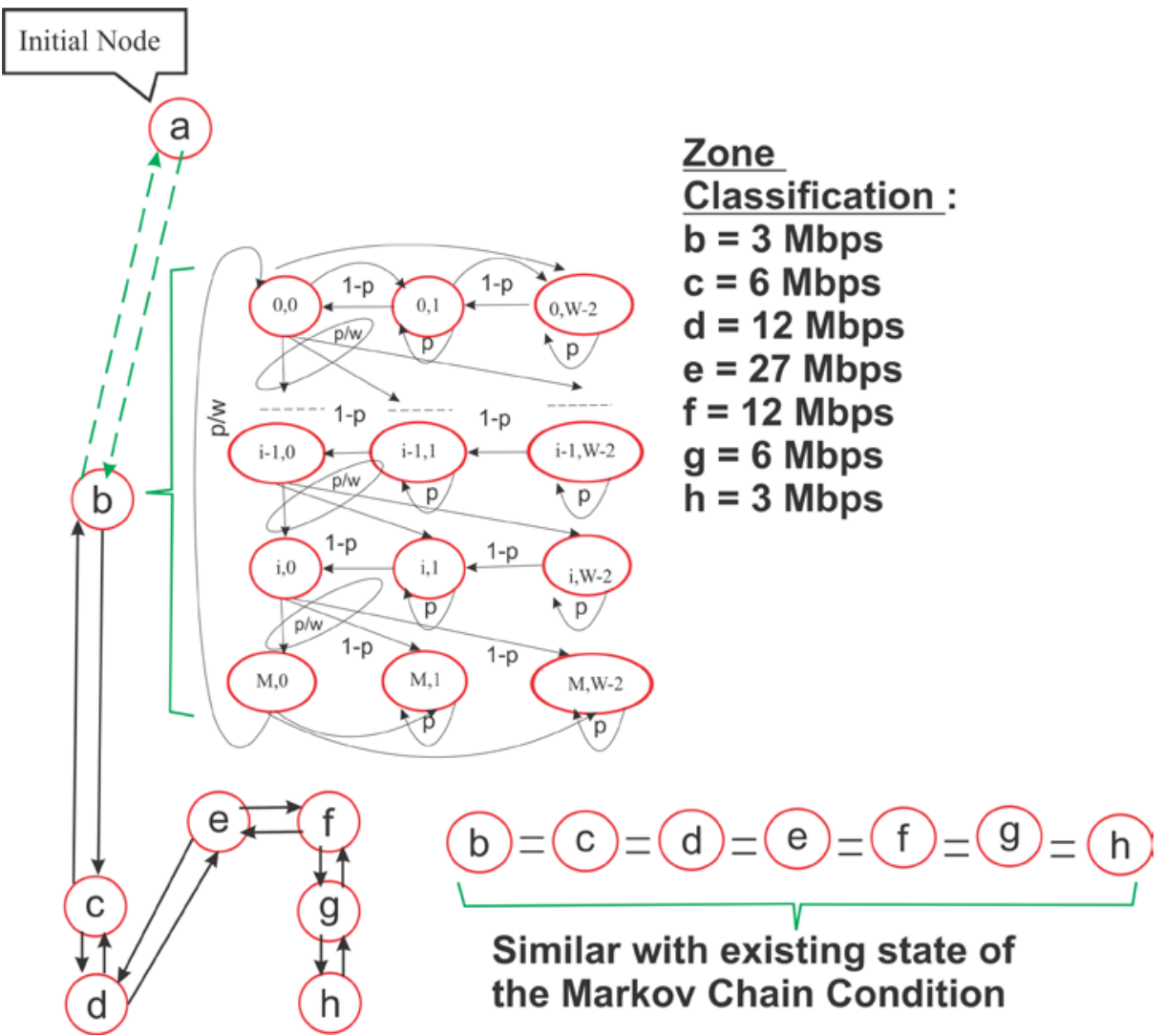

Fig. 2. The proposed of Markov chain model

In Fig. 2, we illustrate our approach to the Markov chain pattern. We used the discrete Markov chain pattern from $[7,8]$ and extended this model by taking the variable data rate and dynamic coordination scheme on the multi-channel MAC of the IEEE 1609.4 standard. In this model, we also define the state for the nodes distribution using the Poisson distribution. We also 
evaluated the Doppler effect using this model. Let $b(t)$ represents the backoff counter in time slot $\mathrm{t}$, and the random process that represents the backoff stage $k$ can be described using notation $s(t)$, where $k \in m$. Then, we can obtain $C W_{\min }$, and $m$ is the maximum backoff stage; therefore, $C W_{\max }=2^{m} W$. Otherwise, we defined $z(t)$ as seven groups of the distribution nodes according to their transmission rate, i.e., 3, 6, 12, 27, 12, 6, and 3 Mbps. We used the transmission rate assumptions based on the maximum bandwidth in the 802.11p standard, i.e., $27 \mathrm{MHz}$. Then, the three-dimensional process $\{\mathrm{s}(\mathrm{t}), \mathrm{b}(\mathrm{t}), \mathrm{z}(\mathrm{t})\}$ can be modelled as a discrete-time Markov chain (DTMC).

In our model, we defined how our model deals with the real environment, called "performance anomaly" [7,8]. Based on the work from [7,8], we found the phenomenon of "performance anomaly" of the IEEE 802.11 standard, which showed startling execution corruption of the stations when utilizing high information rates or those close with the access point (AP). In this work, we evaluate the "performance anomaly" by accounting for the Doppler effect and the dynamic coordination scheme on the multi-channel MAC of the IEEE 1609.4 standard.

We defined one $\mathrm{CCH}$ and six SCH channels in our model. Additionally, we defined the effect of Doppler as the changing in frequency of a wave for the surveyer immigrating relative to its spring. In a vehicular environment, the Doppler effect is caused by high mobility, a dynamic topology, and a trajectory change. In this work, we evaluate the Doppler effect based on the different transmission rates and velocities in each group. We analyse the coordination scheme based on the previous scheme [3-5]. We use the Markov model to evaluate the coordination scheme under the dynamic vehicular traffic condition with high mobility nodes in the multi-channel MAC of the IEEE 1609.4 standard.

We estimate the node distribution in each group using the Poisson and Nakagami fading distribution [11]. Next, according to the work in [11], we define the optimal CW index (CWI) as the optimum initial CW value to conform the adaptive network conditions for better fairness and higher aggregate throughput. We then found the optimal CW index (CWI) based on the distribution node in each group. Finally, we can evaluate the aggregate throughput $S$ for the coordination scheme using the CWI result. We can also optimize the throughput based on the Doppler effect in each group of nodes using the optimum initial CW. The results in [15] showed that the change in the initial CW could increase the throughput.

\subsection{Average Node Distribution}

Next, we calculate the node distribution in each group using the Poisson distribution according to [12]. Firstly, we estimate the communication range under the Nakagami fading distribution.

According to [12], the Nakagami fading distribution considers that the parameters can be adjusted correspond to a variety of empirical measurements. The Nakagami-m appropriation is a likelihood circulation identified with the gamma conveyance. It has a shape measurer and a moment measurer that controls the afloat $\Omega$. The received signal power $x$ is probability density function (pdf) [12,13] can be written as

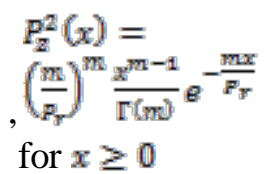

Where $\Gamma\left(\right.$ ) Is the Gamma function; $P_{r}=P_{t} K / r^{\alpha}$ is the average received power; $r$ is the range in meters; $\alpha$ is a path-loss exponent; $K=G_{t} G_{y}\left(C /\left(4 \pi f_{c}\right)\right)^{z} ; C$ is the speed of light; $f_{c}=5.9 \mathrm{GHz}$ is the carrier frequency, $G_{t}$ and $G_{y}$ are a transmitter's and receiver's antennas gain, respectively, $r$, and $m$ is the factor of fading [12]. 
From Eqn. (1), we can figure a cdf of the correspondence extend when the got power is more noteworthy than the limit $P_{\text {th }}$ as

$$
\begin{aligned}
& F_{R}(r)= \\
& 1- \\
& P(x \geq \\
& \left.P_{\text {th }}\right)= \\
& 1- \\
& \int_{P_{\text {th }}}^{m s} P_{z}^{2}(x) d x
\end{aligned}
$$

The average state of a communication area $E[R]$ can be derived as

$E[R]=$

$\int_{0}^{\sin }(1-$

$\left.F_{R}(r)\right) d r$

Substituting (4) into (5) and integrating above the limit, we obtain

$$
E[R]=\frac{1}{\alpha \Gamma(m)} \sum_{i=0}^{m-1} \frac{(m-1) !}{(m-1-1) !} x \Gamma\left(m-1-i+\frac{1}{Q}\right)\left(\frac{m p_{p h}}{p_{\mathrm{r}} K}\right)^{-\frac{1}{\alpha}}
$$

To infer the normal bearer sense run $E\left[L_{c s}\right]$, where hubs can detect the bundle yet can't get it, an indistinguishable methodology from in (6) is taken after

This condition exception is for the received power $P_{C S}$, which defined as the percentage threshold $P_{t h}$ as $P_{C S}=\rho P_{t h}$, where $\rho \in[0,1]$. Therefore, the expected porter sense is

$$
E\left[L_{C S}\right]=\frac{E[R]}{\sqrt[\pi]{\rho}}
$$

We use the same assumptions in [12] for our model analysis to estimate the average node using the Poisson distribution. According to an analytical study [12], we can discover the likelihood of having $N_{\mathscr{e}}$ vehicles inside the scope of any labeled vehicle. The versatility model is reached out to incorporate the base wellbeing between vehicles in every path. This base separation is an arbitrary variables and relies at upon a accompanying vehicle's speed $v_{j}$ if a settled $t_{s}$ is imaginated, which is a reaction time to the driver for respond to the sudden occurrence, which can be described using the single-server queue model, as shown in Fig. 4.

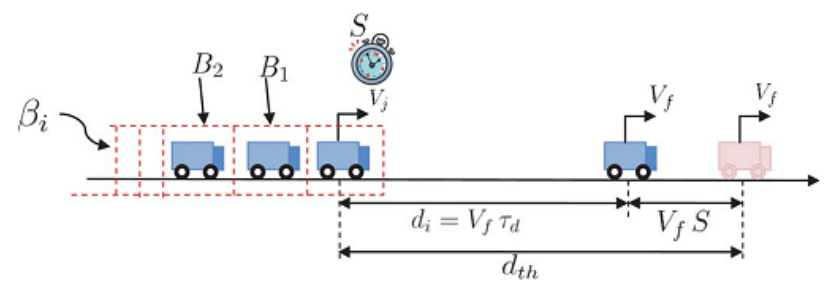

Fig. 3. Single-server queue model [12]

According to [12], when a number of vehicles that across the defined reference point is small, the inter-arrival time $\left(\tau_{d}=1 / \beta_{i}\right)$ between vehicles in the $i^{\text {th }}$ lane is larger than $t_{s}$.

In this issue, the opportunity of having $N_{c i}=k$ vehicles with a communication area of the tagged vehicles (i.e., within a distance of $2 \bar{R}$ ) in the $i^{\text {th }}$ lane is based on the Poisson distribution [12] as

$$
P_{2 R}\left(N_{c i}=k\right)=\frac{\left(\frac{2 \beta_{j} \bar{R}}{\mu}\right)^{k}}{k !} e^{-\frac{2 g_{j} \bar{R}}{\mu}}
$$


and the average number of vehicles about the tagged vehicle in a ith lane

$$
N_{c i}=\frac{2 \beta_{i} R}{\mu}
$$

Based on [11], the optimal contention window (CW) is subject to the dynamic hub number in the system $\left(N_{c i}\right)$. Therefore, in the next section, we define the optimal contention window $(\mathrm{CW})$.

\subsection{Optimal CW Index (CWI)}

Next, we obtain the optimal CW index (CWI) based on [11] and extend this approach by using the variable data rate based on the nodes distribution and the Doppler effect. The optimal CW index (CWI) is an optimum initial CW value to adjust the dynamic system conditions for better transient decency and higher total throughput [11]. Finally, we can evaluate the aggregate throughput $\mathrm{S}$ for the coordination scheme using the CWI result. Firstly, we define the aggregate throughput $\mathrm{S}$ as [11]

$S=\frac{E\lfloor P\rfloor+F_{e d} \cdot\left(T_{D a t a} / T_{S}\right) \cdot \lambda \cdot R_{\text {Data }}(t)}{E[D]}$

where $F_{g d}=p_{s} \cdot T_{s} \sum_{\mathrm{i}=0}^{K-1} \sum_{j=0}^{\mathrm{i}} p^{\mathrm{i}} E\left[U^{[j]}\right]$ is the time that different hubs transmit effectively aside from the labeled hub amid the mean defer time. $E[P]$ and $R_{D a t a}(t)$ are the normal bundle payload lengths and information rates, separately. $\lambda$ is the proportion of the payload size to full bundle length with the header messages.

The throughput $S$ in the Eqn. (8) can be further simplified as in Eqn. [15]

$$
\begin{aligned}
& S=\frac{p_{S} \cdot T_{S} \sum_{i=0}^{K-1} \sum_{j=0}^{\mathrm{i}} p^{\mathrm{i}} E\left[U^{[j]}\right] \cdot\left(T_{\text {Data }} / T_{S}\right) \cdot \lambda \cdot R_{\text {Data }}(t)}{E[D]}+\frac{E[P]}{E[D]} \\
& S=\frac{E[P]\left(1+p_{S} \sum_{i=0}^{K-1} \sum_{j=0}^{\mathrm{i}} p^{\mathrm{i}} E\left[U^{[j]}\right]\right)}{E[D]}
\end{aligned}
$$

Based on [11], the optimal $C W$ can receive a optimum throughput with a node number of $N$. According to [11], the network delay is modelled more accurately when the new greateful transmission time $T_{S}$ and collision time $T_{C}$ are calculated:

$\left\{\begin{array}{l}T_{S}=T_{\text {RTS }}+3 T_{\text {SIFS }}+4 \sigma+T_{\text {CTS }}+T_{\text {DATA }}+T_{\text {head }}+T_{A C K} \\ T_{C}=T_{\text {RTS }}+T_{\text {SIFS }}+2 \sigma+T_{\text {DIFS }}\end{array}\right.$

where $T_{\text {DATA }}$ and $T_{\text {head }}$ speak to the transmission duration of the MAC edge and header of the physical layer, separately. $\sigma$ and $T_{\text {SIFS }}$ are the propagation delay and the SIFS duration. $T_{R T S}$, $T_{C T S}$, and $T_{A C K}$ denote the transmission duration of an RTS, CTS, and ACK frames [11].

According to Eqn. (8), we can simplify Eqn. (10) using $C W$ and $\partial S / \partial C W=0$ and then neglecting the stuffs that is less than or equals to the third-order stuff $1 / \mathrm{CW}^{\mathrm{a}}$ 


$$
\begin{aligned}
& \partial S / \partial C W=\frac{N \cdot T_{\text {SLOT }}}{2} \cdot C W^{2}+2(N-1)^{2} \cdot T_{\text {SLOT }} \cdot C W \\
& \quad+(N-1)\left(4 T_{\text {SLOT }}-2 T_{C}-2 T_{\text {EISS }}\right) N^{2} \\
& \quad+(N-1)\left(2 T_{\text {EISS }}-2 T_{\text {DIFS }}-14 T_{\text {SLOT }}\right) N \\
& \quad+(N-1)\left(2 T_{\text {DIFS }}+10 T_{\text {SLOT }}\right) N
\end{aligned}
$$

We can obtain the optimal CW by solving Eqn. (11) as

$$
C W=\frac{\sqrt{2 N(N-1) T_{S L O T}\left[T_{E C S}(N-1)^{2}+T_{1}\left(N-\frac{T_{2}}{T_{1}}\right)\right]}}{N \cdot T_{\text {SLOT }}}+\frac{-2(N-1)^{2} T_{\text {SLOT }}}{N \cdot T_{\text {SLOT }}}
$$

where $T_{1}=2 T_{\text {EIFS }}+4 T_{C}+2 T_{\text {DIFS }}+4 T_{\text {SIOT }}, T_{2}=T_{1}-$ $2 T_{C}-2 T_{S L O T}$, and $T_{E C S}=2 T_{E I F S}+2 T_{C}-2 T_{S L O T}$

Consequently, we can calculate the result of the $\mathrm{CW}$ from (12)

$C W \approx\left(\sqrt{\frac{2 T_{E C S}}{T_{S L O T}}}-2\right) \cdot(N-1) \approx \theta \cdot(N-1)$

where $\theta=\left(\sqrt{\frac{2 T_{R C S}}{T_{S L O T}}}-2\right)$ and is referred to as the $C W$ Index

(CW I).

\subsection{Throughput Analysis}

We use the three-dimensional Markov chain model to evaluate and analyse the throughput. First, according to the work in [3-5], we can obtain the probability of successful transmission $P_{\text {ouc }}$, where a channel collision occurs with a probability of $P_{\text {col }}$ or the channel is idle with a probability of $P_{\text {idle }}$

$$
\left\{\begin{array}{l}
P_{\text {idllg }}=(1-\tau)^{n} \\
P_{\text {busy }}=1-P_{\text {iddls }}=1-(1-\tau)^{n} \\
P_{\text {swe }}=n \pi(1-\tau)^{n-1} \\
P_{\text {col }}=P_{\text {busy }}-P_{\text {sus }}=1-(1-\tau)^{n}-n \tau(1-\tau)^{n-1}
\end{array}\right.
$$

According to [10], $w_{\mathrm{i}, k}$ denotes the backoff window for a station in gathering $\mathrm{i}$ at backoff arrange $\mathrm{k}$. In the routine DCF instrument utilizing bearer sense numerous entrance with impact shirking (CSMA/CA), all stations in any gathering i have a similar starting (at backoff organize 0 ) backoff window $w_{\mathrm{i}, 0}$ for all $i=1,2,3,4,5$, 6, and 7 [10].

We use the same assumptions with [10] at the point when the channel is sit out of gear amid the DCF interframe space (DIFS), where every station chooses an arbitrary backoff counter among $\left[0, w_{\mathrm{i}, 0}-1\right]$ slots and sits tight for the comparing number of spaces before endeavoring to get to the remote medium. The estimation of the backoff counter is decremented by one at whatever point an opening is detected as sit. On the off chance that there are transmissions from different stations amid this period, the backoff counter commencement is suspended [10]. At that point, when the channel gets to be distinctly sit without moving, the station continues its backoff procedure after a DIFS sit out of gear period 
[10]. At the point when the backoff counter esteem achieves zero, the station transmits the pending edge [10].

Then, we evaluate the transition state probability in figure 3 . We have seven groups of nodes according to their transmission rate, i.e., 3, 6, 12, 27, 12, 6, and 3 Mbps. Therefore, we define $z(t)$ that represents the group of nodes 1 , where $1 \leq i \leq N$. In this paper, we rework the probability of successful transmission $P_{\text {sue }}$, where a channel collision occurs with the probability of $P_{\text {coll }}$ or the channel is idle with a probability of $P_{\text {idle }}$. The state of the Markov chain is denoted as $\left(i, k_{v},\right)_{v} 1 \leq i \leq N_{v} 0 \leq k \leq m_{v} 0 \leq l \leq w_{i, k}-1$, where $i_{v} k_{s}$ and $l$ represent group $i$, the backoff stage $k$, and the backoff counter value $l$, respectively. Let $t_{z}$ represent the conditional transmission probability of nodes in zone $\mathrm{z}$ as $\mathrm{N}$ group zones. We define $\mathrm{m}$ as the maximum backoff stage. Additionally, $W_{\max }$ represents the maximum contention window, and $W_{z}$ represents the minimum contention window size $\mathrm{CW}_{\min }$ associated in zone $\mathrm{z}$. We define $\mathrm{E}[\mathrm{X}]$ as the mean time of transmission data of the tagged point in area $z$. We define $\mathrm{E}\left[\mathrm{Tx}_{\mathrm{suc} z}\right]$ as the mean duration of successful transmission of the tagged point in area z. Furthermore, we derived from [10] by considering the Doppler effect :

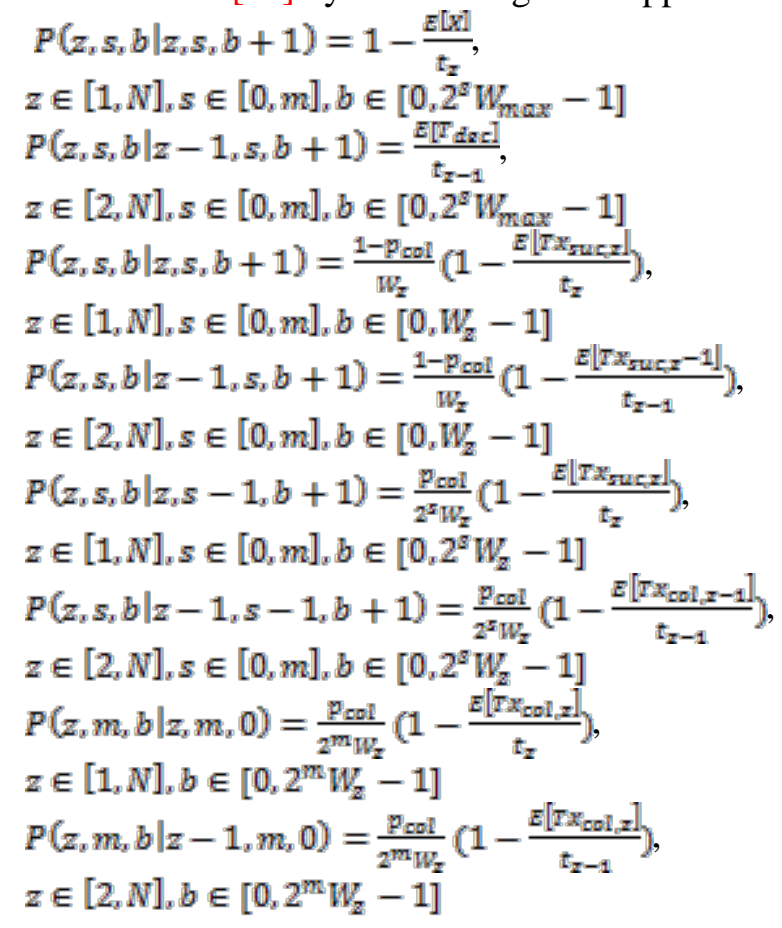

An unsuccessful (re)transmission attempt can occur due to collision of this station with at least one of the $n-1$ remaining stations, which has the probability of $p_{\text {col }}[14]$ :

$$
p_{\text {col }}=1-\prod_{z=1}^{N}(1-\tau)^{n}-n \tau(1-\tau)^{n-1}
$$

An error frame occurs with probability $P_{\varepsilon}$ due to the channel fading and/or noise. Because both events are independent, the probability of node collision in each zone $i, p_{e, i}$ can be expressed as $[10,14]$

$$
\begin{aligned}
& p=p_{c, i}=1-\left(1-p_{c o i}\right)\left(1-P_{\theta}\right) \\
& =p_{c o l}+P_{\theta}-p_{c o l} P_{\theta} \\
& \text { where } P_{\theta}=1-\left(1-P_{g}\right)^{L} \text { and } P_{g}=E\left[\mathrm{P}_{b}(\gamma)\right]
\end{aligned}
$$


$P_{\theta}=\mathrm{R}_{\mathrm{b}}(\gamma)= \begin{cases}\mathrm{P}_{2}=\mathrm{Q}(\sqrt{2 \gamma(\mathrm{k})} & \text { for BPSK } \\ \mathrm{P}_{4}=2 \mathrm{Q}(\sqrt{\gamma(\mathrm{k})})-\mathrm{Q}^{2} \sqrt{\gamma(\mathrm{k})}, & \text { for QPSK }\end{cases}$

Next, we determine $\tau$ as the transmission probability where a station conducts the success transmission at the slot. By using the random distribution node and considering the AWGN, we calculate the transmission probability $\tau$ of each station.

Because transmission occurs only in states $(k, 0)$, the probability $\tau$ of a station transmitting in a random chosen slot able be showed as

$$
\begin{aligned}
& \tau=\sum_{k=0}^{m+f} b_{i, 0}=\sum_{k=0}^{m+f} p^{k} \cdot b_{i, 0,0}=\frac{1-p^{m+f+1}}{1-p} \cdot b_{i, 0,0} \\
& \text { Then, probability } b_{i, k i l} \text { for } 0<i \leq(m+f) \text { can be given simply as } \\
& b_{i, k, l}=\lim _{t \rightarrow \infty} P\{U(i, t)=k, C(i, t)=l\} \\
& b_{i, k, l}=p_{c, i}^{k} b_{i, 0,0}, \quad 0 \leq k \leq m+f \\
& b_{i, k_{i}}=\left(\frac{W_{i, k}-1}{W_{i, k}}\right) \frac{1}{1-\left(p_{c i} i\right.} b_{i, k_{i}, 0}, 0 \leq k \leq m+f \text {. } \\
& 1 \leq l \leq w_{\mathrm{i}, k}-1
\end{aligned}
$$

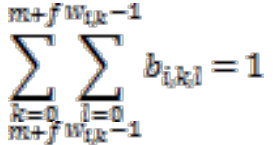

$$
\begin{aligned}
& \sum_{k=0} \sum_{i=0} b_{i, k i}=1 \\
& =\sum_{k=0}^{m-1} b_{i, k, 0} \cdot p_{c, i}{ }^{k}+b_{i, m_{0}, 0}+\sum_{k=0}^{m+f} \sum_{i=0}^{W_{i, l^{-1}}}\left(\frac{W_{i, k}-l}{W_{i, k}}\right) \frac{1}{1-\left(p_{c, i}\right)} b_{i, k, 0} \\
& b(i, 0,0)=\left(\frac{d_{z}}{\sum_{z=1}^{n} d n}\right) \frac{2\left(1-p_{c i}^{m+f+1}\right)\left(1-2 p_{c i}\right)}{\left(1-p_{c i}{ }^{m+f+1}\right)\left(1-2 p_{c i}\right)+\left(1-2 p_{c i}\right)} \\
& +W \cdot\left[1-\left(2 p_{c i}\right)^{m+1}\right]\left(1-\left(p_{c i}\right)\right) \\
& +p_{c i}\left(2 p_{c i}\right)^{m}\left(1-p_{c i f} f\right)\left(1-2 p_{c i}\right)
\end{aligned}
$$

Then, we can obtain the probability $\tau$ as

$$
\begin{gathered}
\tau_{i}=\frac{\sum_{i=0}^{\mathbb{N}} b(i, k, 0)}{\left(\frac{d_{z}}{\sum_{z=1}^{n} d n}\right)}=\frac{\sum_{i=0}^{\mathbb{N}}\left(p_{c, i}\right)^{k} b(i, 0,0)}{\left(\frac{d_{z}}{\sum_{z=1}^{n} d n}\right)} \\
\tau_{i}=\left(\frac{\left(1-\left(p_{c i}\right)^{k+1}\right)}{\left(1-p_{c_{i} i}\right)}\right)\left(\frac{b\left(i_{i}, 0\right)}{\left(\frac{d_{z}}{\sum_{z=1}^{n} d n}\right)}\right)
\end{gathered}
$$

Combining the equation above, we obtain $\tau_{i}$ :

$$
\begin{aligned}
& \tau=\frac{2\left(1-p^{R+f+1}\right)(1-2 p)(1-p)}{\left(1-p^{R+f+1}\right)(1-2 p)\left[\left(1-p^{R+f+1}\right)-(1-p)\left(1-p^{R+1}\right)\right]} \\
& +(1-p)\left[w\left[1-(2 p)^{R+1}\right](1-p)+p(2 p)^{R}(1-p f)(1-2 p)\right]
\end{aligned}
$$


Next, we define $\mathrm{P}_{\mathrm{tr}}$ as the opportunity of at least one point in transmission in the cognationed duration, and $P_{s}$ is the symbol error rate (SER). In this work, we use the same assumptions with [14] by taking the frame error probability $P_{\Theta}$ into the calculation.

$$
\left\{\begin{array}{l}
\mathrm{P}_{\text {tr }}=1-P_{\text {iddllg }}=1-(1-\tau)^{n} \\
P_{f}=1-\left(1-p_{\text {col }}\right)\left(1-P_{\mathrm{g}}\right) \\
P_{s}=\frac{1+\bar{\gamma}(1-\rho)}{2(1+\bar{\gamma})}
\end{array}\right.
$$

Moreover, the saturated throughput in each zone is obtained using [15], which is the average payload of information that able be transmitted in a duration slot on a SCH channel by considering the effect of bit error, and the Doppler effect can be calculated by the following formula.

$$
\begin{aligned}
& S=\frac{E[\text { payload information transmitted in a slot time }]}{E[\text { length of slot time }]} \\
& S=\frac{P_{g} P_{t r}\left(1-P_{f}\right) E[L]}{\left(1-P_{t r}\right) \sigma+P_{t v} P_{g}\left(1-P_{f}\right) T_{g}+P_{t r}\left(1-P_{g}\right) T_{c}+P_{t r} P_{g} P_{f} T_{\theta}}
\end{aligned}
$$

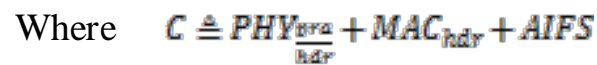

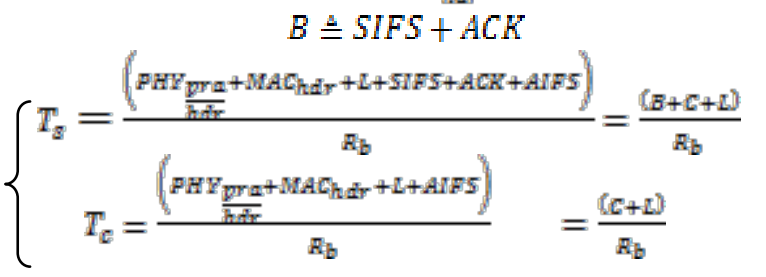

$\gamma=\Omega E_{B} / N_{0}$ is the fading of average SNR, $\Omega$ is the gain of channel, and $\rho$ is the fading correction parameter, where $\rho=J_{0}\left(2 \pi f_{d} T_{b}\right)$. Additionally, $f_{d}$ denotes the Doppler spread.

$$
f_{d}=\left(\frac{c+v_{f}}{c+v_{g}}\right) f_{0}
$$

$c$ represents the velocity of waves in the medium.

$v_{\gamma}$ represents the velocity of the receiver relative to the medium.

$v_{s}$ represents the velocity of the source relative to the medium.

$f_{0}$ denotes the source frequency $\left(f_{0}=5.9 \mathrm{GHz}\right)$.

From Equations (19), (20), (21), and (24), we can obtain the formula of the saturated throughput as follows:

$$
S T=\frac{N_{x}\left(1-\left(1+\gamma\left[1-J_{b}\left(2 \pi f_{d} T_{b}\right)\right] / 2(1+\gamma)\right)\right)^{L} L R_{b}}{(1-\tau) \sigma R_{b}+N_{x}(B+C+L)+\left[(1-\tau)^{-W+2}-(1-\tau)-N_{x}\right](C+L)}
$$

Finally, the aggregate throughput can be defined from [15] as the following:

$$
\begin{aligned}
& A T= \\
& \sum_{i=1}^{N}\left(S T_{i} \times\right. \\
& \left.r_{i}\right)
\end{aligned}
$$

Based on Luo [17], the effect of gain is more urgent in IEEE 802.11p because the velocity. In 802.11p systems, when a 10-MHz bandwidth is brought, the Doppler spectrum with velocities 
of $10.8 \mathrm{~km} / \mathrm{h}$ and $216 \mathrm{~km} / \mathrm{h}$ is approximately $59 \mathrm{~Hz}$ and $1.18 \mathrm{kHz}$, respectively. In this work, the simulated velocities of the vehicles are between $80-120 \mathrm{~km} / \mathrm{h}$.

In the next section, we evaluate and simulate the coordination scheme, which considers the Doppler effect.

\section{Performance Evaluation}

Using MATLAB, we evaluate the performance of the Markov model for the coordination scheme based on the IEEE 1609.4 standard. We simulate the scenario with the 119 vehicle nodes, the velocities of the vehicles are between $80-120 \mathrm{~km} / \mathrm{h}$, the packet length is 2000 bytes, and there are seven groups of distributed nodes according to their transmission rate, i.e., 3, 6, 12, 27, 12, 6, and 3 Mbps. The channel configuration uses the variable value for the control and administration channel interims. The protect interim esteem is $4 \mathrm{~ms}$. Table I shows every one of the parameters utilized as a part of our recreation. While certain parameters remain settled, others are fluctuated to watch any changed conduct of the system.

Table 1. Simulation Parameters

\begin{tabular}{|l|l|}
\hline \multicolumn{1}{|c|}{ Parameters } & Values \\
\hline Number of CCH & 1 \\
\hline Number of SCHs & 4 \\
\hline Group of nodes & 7 \\
\hline Service packet length & 2000 bytes \\
\hline$C W_{\min }$ & 32 \\
\hline$C W_{\max }$ & 1024 \\
\hline WSA/RFS & $\begin{array}{l}160 \quad \text { bits }+ \text { PHY } \\
\text { header }\end{array}$ \\
\hline ACK & $\begin{array}{l}112 \text { bits }+ \text { PHY } \\
\text { header }\end{array}$ \\
\hline Slot time & $20 \mu \mathrm{s}$ \\
\hline SIFS & $10 \mu \mathrm{s}$ \\
\hline DIFS & $50 \mu \mathrm{s}$ \\
\hline Number of vehicles & 119 nodes \\
\hline Velocity of vehicles & $80-120 \mathrm{~km} / \mathrm{h}$ \\
\hline
\end{tabular}

\subsection{Performance Evaluation of the Probability of WSA Packet Transmission}

We use the three-dimensional Markov chain model to determine the probability of the WSA packet transmission that is affected by the reservation channel CCH contention models and the anomalous performance due to the different transmission rates in each zone. The probability of the WSA packet transmission is calculated based on two variables, i.e., the transmission rate and the number of distribution nodes in each zone. The influence of these two variables on the probability of the WSA packet transmission on the DCF model were simulated using MATLAB. In this work, we have different transmission rates, i.e., 3, 6, 12, 27, 12, 6, and 3 Mbps. We also consider the Nakagami propagation model to analyse the performance results.

Fig. 4 shows the probability of WSA packet transmission with the optimum initial contention window (CW) based on the nodes distribution in each zone, where the probability of the WSA packet transmission is better than that without determining the optimum initial contention window $(\mathrm{CW})$. 
We found that the probability of WSA packet transmission with the optimum initial contention window (CW) enhanced the performance results with a mean of approximately $24.67 \%$.

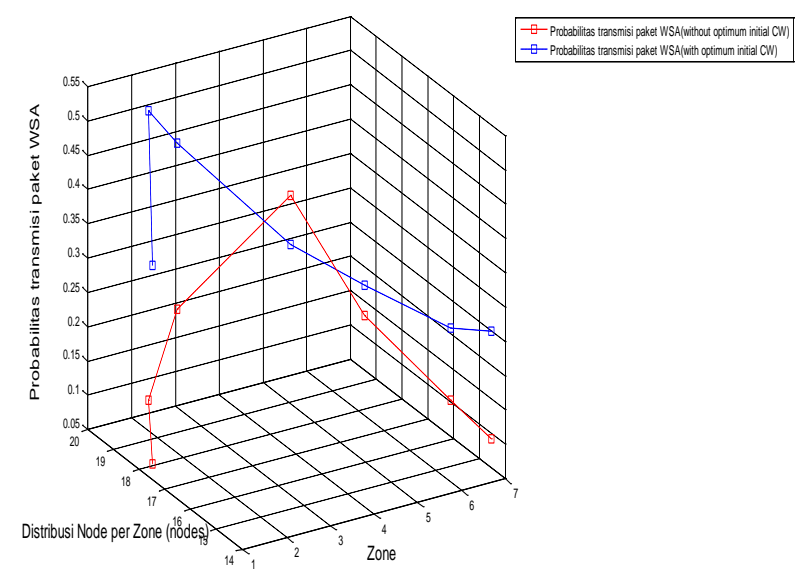

Fig. 4. Probability of WSA packet transmission

\subsection{Performance Evaluation of the Average Transmission Delay of Service Packets}

In this work, we evaluate the analytical model for the average transmission delay

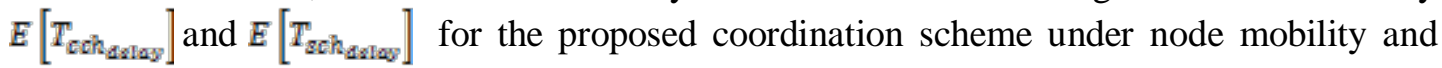
frame error caused by AWGN. In our model, different transmission rates were used, i.e., 3, 6, 12, 27, 12, 6, and 3 Mbps. We also consider the Nakagami propagation model to analyse the performance results. It was assumed that the node had the highest data rates on a certain channel with service packet lengths of up to 2000 bytes.

By using [3-5], we can obtain $E\left[T_{\text {cch }_{\text {dulasy }}}\right]$ and $E\left[T_{\text {seh }_{\text {Aslaw }}}\right]$ as

$$
\begin{aligned}
& E\left[T_{\text {rech_delay }}\right]=\frac{1}{2}\left(G_{1}+1\right) . E[X]
\end{aligned}
$$

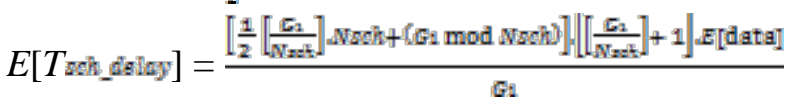

where $G_{1}$ shows the number of reservations made on the CCH amid the WSA interim, and $G_{2}$ is the quantity of administration bundles transmitted on all Nsch SCHs amid the SCH interim [3-5].

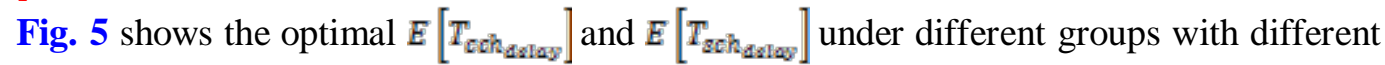
transmission rates and node velocities in each group. We also evaluated service packets based on the frame error caused by the AWGN. We found that $E\left[T_{\text {coch }}\right.$ aslay $\left._{\text {ay }}\right]$ increases correspondingly with increases in the transmission rate and node velocity. The average delay $E\left[T_{\operatorname{sch}_{\mathbb{A s l a w}}}\right]$ significantly increases, which is caused by the distribution node in each group.

Moreover, the frame error caused by the AWGN influences the service packet data and will

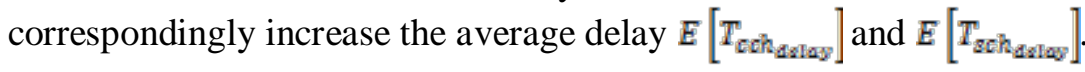




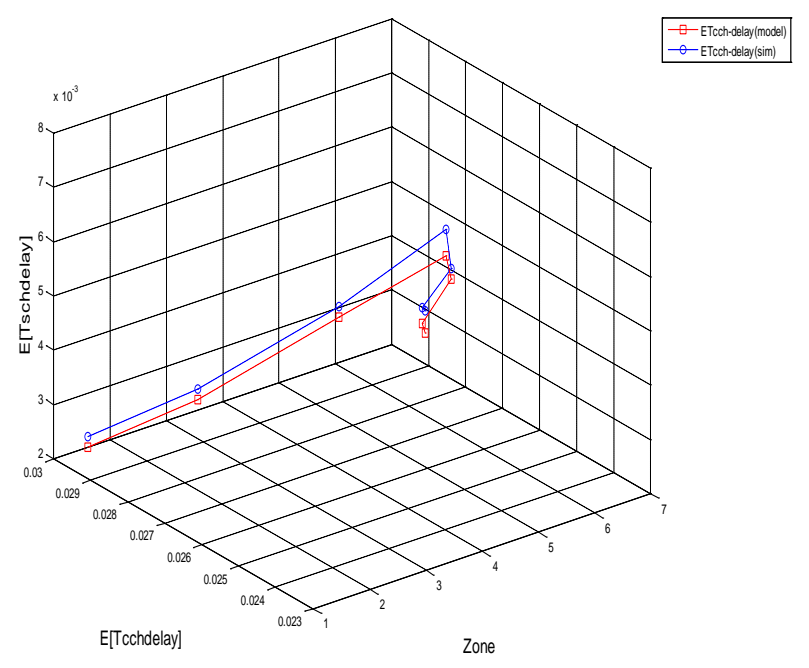

Fig. 5. Average delay transmission under different groups

\subsection{Performance Evaluation of the Average Transmission Delay of Service Packets}

To analyse and perform the saturated throughput on the SCHs, a three-dimensional Markov chain model was used. In this work, we used different transmission rates in each groups, i.e., 3, 6, 12, 27, 12, 6, and 3 Mbps. We also considered the error frame caused by the AWGN. In this work, we used the Nakagami propagation model to analyse the saturated throughput. We also used the same assumptions as those in [6-8] for the saturated throughput on the SCHs caused by the SCH interval, the mean interval that transmits a service packet on $\mathrm{SCH}$, the number of SCHs, and the payload of the service packet. In this model, we also considered the different transmission rates and node velocity in each group to analyse the saturated throughput on the SCHs.

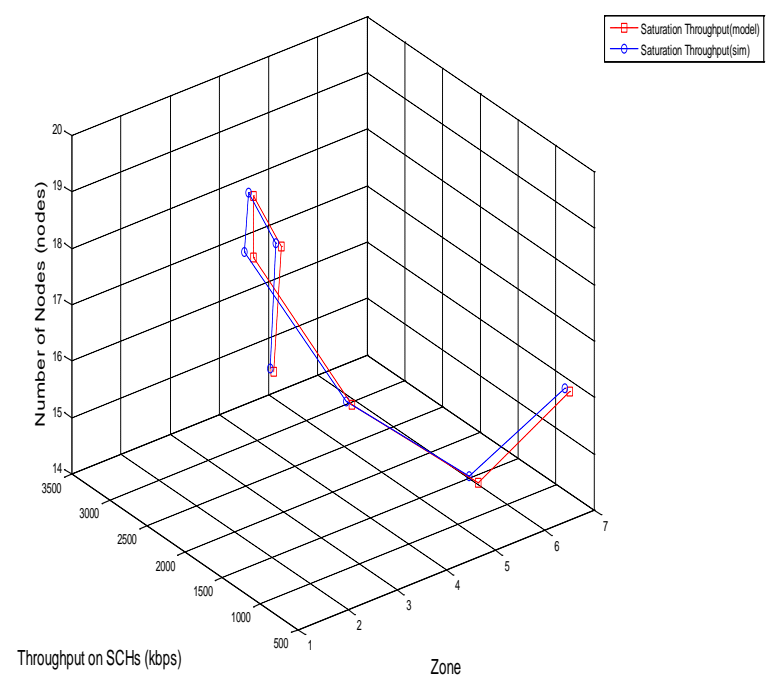

Fig. 6. Saturated throughput on SCHs under different groups 
Fig. 6 shows the optimum saturated throughput on SCHs that considered the error frame caused by the AWGN noise. We found that the saturated throughput on the SCHs reduce correspondingly as the error frame increases, which is caused by the AWGN. Moreover, as the transmission rate and node velocity increase, the saturated throughput on the SCHs also increases.

\section{Conclusion and Future Work}

We evaluated the performance of the dynamic channel coordination scheme that is affected by the high mobility node and additive white Gaussian noise (AWGN). We found that the probability of the WSA packet transmission with the optimum initial contention window (CW) based on the nodes distribution in each zone is better than that without determining the optimum initial contention window (CW). We found that the probability of the WSA packet transmission with the optimum initial contention window (CW) enhanced the performance results with a mean of approximately $24.67 \%$.

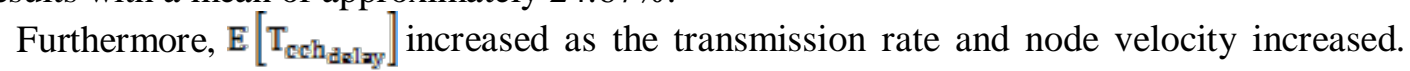

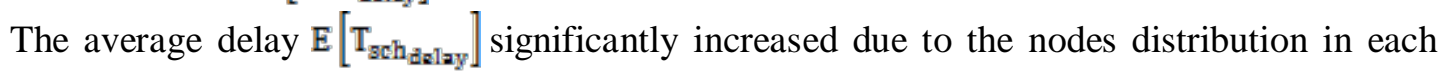
group. Moreover, the frame error caused by the AWGN influenced the service packet data and correspondingly increased the average delay $\mathrm{E}\left[\mathrm{T}_{\text {cch }_{\text {dalay }}}\right]$ and $\mathrm{E}\left[\mathrm{T}_{\text {sch }}{ }_{\text {đalay }}\right]$.

Additionally, the saturated throughput is influenced by the increase in the transmission rate and node velocity. Similar to the average delay, the frame error caused by the AWGN influenced the service packet data, which corresponds with the saturated throughput on the SCHs.

Later on, we plan to augment the 3D Markov model evaluation with the influence of the hidden nodes problem in relation to the node mobility performance on the IEEE 1690.4 standard.

\section{References}

[1] Hongseok Yoo and Dongkyun Kim, "Dynamic Channel Coordination Schemes for IEEE 802.11p/1609 Vehicular Networks : A Survey," International Journal of Distributed Sensor Networks, Volume 2013, Hindawi Publishing, 2013. Article (CrossRef Link)

[2] IEEE Std 1609.4-2010, IEEE Standard for Wireless Access in Vehicular Environments (WAVE) Multichannel Operation, IEEE, 2011. Article (CrossRef Link)

[3] Q. Wang, S. Leng, H. Fu, and Y. Zhang, "An IEEE 802. 11p-based multichannel MAC scheme with channel coordination for vehicular Ad Hoc networks," IEEE Transactions on Intelligent Transportation Systems, vol. 2013, no. 2, pp. 449-458, 2012.

Article (CrossRef Link)

[4] Q. Wang, S. Leng, H. Fu, Y. Zhang, and H. Weerasinghe, "An enhanced multi-channel MAC for the IEEE 1609.4 based vehicular Ad Hoc networks," in Proc. of the IEEE International Conference on Computer Communications (INFOCOM '10), San Diego, Calif, USA, March 2010. Article (CrossRef Link)

[5] Q. Wang, S. Leng, Y. Zhang, and H. Fu, "A QoS supported multi-channel MAC for vehicular Ad Hoc networks," in Proc. of the IEEE 73rd Vehicular Technology Conference (VTC '11), Budapest, Hungary, May 2011. Article (CrossRef Link)

[6] J. Mo, H. So, and J. Warland, "Comparison of Multi-channel MAC protocols," IEEE Trans. Mobile Comput., vol. 7, no. 1, pp. 50-65, Jan 2008. Article (CrossRef Link)

[7] D. Zhu and D. Zhu, "Performance analysis of A multi-channel MAC with Dynamic CCH interval in WAVE system," in Proc. of the 2nd International Conference On Systems Engineering and Model, Beijing, China, April 2013. Article (CrossRef Link) 
[8] N. Lu, Y. S. Ji, F. Q. Liu, and X. H. Wang, “A dedicated multi-channel MAC protocol design for VANET with adaptive broadcasting,” in Proc. of WCNC, pp. 1-6, 2010.

Article (CrossRef Link)

[9] Duck-Yong Yang, Tae-Jin Lee, Jin Bong Chang, and Sunghyun Choi, "Performance Enhancement of Multirate IEEE 802.11 WLANs with Geographically Scattered Stations,” IEEE Transactios on Mobile Computing, Vol. 5, No.7, July 2006. Article (CrossRef Link)

[10] Tom H. Luan, Xinhua Ling, and Xuemin (Suherman) Shen, "MAC in Motion : Impact of Mobility on the MAC of Drive-Thru Internet,” IEEE Trans. Mobile Computing, vol. 11, no. 2, pp. 305-319, February 2012. Article (CrossRef Link)

[11] Shi Chun, Dai Xianhua, Liang Pigyuan, and Zhang Han “Adaptive Access Mechanism with Optimal Contention Window Based on Node Estimation Using Multiple Theresholds,” IEEE Transactions Wireless Communicatios, Vol. 11, No. 6, June 2012. Article (CrossRef Link)

[12] Khalid Abdel Hafeez, Lian Zhao, Bobby Ma, and Jon W. Mark, "Performance Analysis and Enhancement of the DSRC for VANET's Safety Applications," IEEE Transactions on Vehicular Technology, Vol. 62, No. 7, September 2013. Article (CrossRef Link)

[13] He J., Tang Z., Yang Z., Cheng W., Chou C.T, “ Performance Evaluation of Distributed Access Scheme in Error-Prone Channel, in Proc. of IEEE TENCON 2002, pp. 1142-1145, October 2002. Article (CrossRef Link)

[14] Bianchi, G.; "Performance analysis of the IEEE 802.11 distributed coordination function," Selected Areas in Communications, IEEE Journal on , vol.18, no.3, pp.535-547, Mar 2000. Article (CrossRef Link)

[15] Doan Perdana and Riri Fitri Sari, "Mobility Models Performance Analysis using Random Dijkstra Algorithm and Doppler Effect for IEEE 1609.4 Standard,” International Journal of Simulation, Systems, Science, and Technology, United Kingdom Simulation Society Article (CrossRef Link)

[16] Luo, T., Wen, Z., Li. J, Chen, H.H, "Saturation Throughput Analysis of WAVE Networks in Doppler Spread Scenarios IET Communcations," special on Vehicular Ad Hoc and Sensor Networks, September 2009. Article (CrossRef Link) 


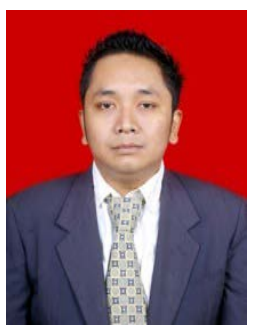

Doan Perdana received his BSc and MSc degrees in Telecommunication Engineering, from the Institute of Technology Telkom in 2004 and 2012, respectively. He is currently pursuing his doctorate in the Electrical Engineering Department, University of Indonesia. His interests include Telecommunication Systems and Computer Engineering.

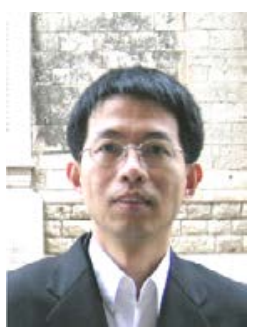

Ray-Guang Cheng $\mathrm{PhD}$ is a Professor of Electronic and Computer Engineering of National Taiwan University Science and Technology. He received his B.E, M.E, and PhD degrees in Communication Engineering from National Chiao-Tung University, Taiwan, ROC, in 1991, 1993, and 1996, respectively. His current research interests include machine-to-machine communications, multi-hop cellular networks, and network architecture for radio over fibres.

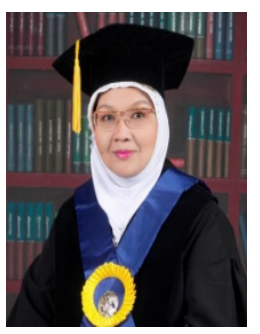

Riri Fitri Sari $\mathrm{PhD}$ is a Professor of Computer Engineering in the Electrical Engineering Department of Universitas Indonesia. She received her BSc degree in Electrical Engineering from Universitas Indonesia. She completed her MSc in Software System and Parallel Processing from the University of Sheffeld, UK. She was awarded her PhD in Computer Science from the University of Leeds, UK. Riri Fitri Sari is a senior member of the Institute of Electrical and Electronic Engineers (IEEE). 